\title{
Specification of axial identity in the mouse: role of the Hoxa-5 (Hox1.3) gene
}

\author{
Lucie Jeannotte, ${ }^{1,2,6}$ Margot Lemieux, ${ }^{2}$ Jean Charron, ${ }^{2}$ Françoise Poirier, ${ }^{1,3}$ \\ and Elizabeth J. Robertson ${ }^{1,4,5}$
}

\begin{abstract}
${ }^{1}$ Department of Genetics and Development, Columbia University, New York, New York 10032 USA; ${ }^{2}$ Centre de Recherche en Cancérologie de l'Université Laval, Hôtel-Dieu de Québec, Québec, Canada, G1R 2J6; ${ }^{3}$ Institut National de la Santé et de la Recherche Médicale (INSERM) U. 257, CHU Cochin, 75014 Paris, France; ${ }^{4}$ Departments of Cellular and Developmental Biology, and Biochemistry and Molecular Biology, Harvard University, Cambridge, Massachusetts 02138 USA; ${ }^{5}$ aymond and Beverly Sackler Foundation, New York, New York 10032 USA
\end{abstract}

\begin{abstract}
Numerous lines of study have suggested that the Hox genes, encoding putative transcription factors, are key genes in the establishment of the body plan of the mammalian embryo. To examine the role of Hoxa-5 (Hox1.3) gene during development, we have used targeted mutagenesis in embryonic stem cells to produce a strain of mice carrying a disrupted Hoxa-5 allele. The viability of homozygous mutant mice is markedly reduced, with $50 \%$ of the mutant animals dying at birth or shortly thereafter. Analysis of the skeleton of Hoxa-5 mutants reveals a number of homeotic transformations restricted to the cervical and thoracic regions. Of these, one of the most frequent morphological abnormalities is the posterior transformation of the seventh cervical vertebra into the likeness of a thoracic vertebra complete with a pair of ribs. These results demonstrate that the Hoxa-5 gene has an important role in the establishment of the skeleton during development and contributes to the process whereby the axial structures are determined.
\end{abstract}

[Key Words: Gene disruption; Hox gene; axial identity; homeotic transformation]

Received July 14, 1993; revised version accepted August 26, 1993.

Embryonic development can be viewed as being controlled by the expression of a hierarchy of regulatory genes. In Drosophila, a critical position in the developmental hierarchy is occupied by the homeotic genes (Akam 1987). These genes are thought to control the formation of body segment-specific structures by regulating the expression of specific sets of downstream effectors that, in turn, direct the morphogenetic events leading to the formation of complex body forms. Consistent with this notion, mutations in homeotic genes result in alterations to segmental identities (Lewis 1978).

Hybridization analysis has revealed the presence of numerous homeo box-containing genes in the genomes of vertebrates. In human and mouse, the 38 homeo box genes of the Antennapedia (Antp) class identified so far are arranged in four gene clusters located on different chromosomes (McGinnis and Krumlauf 1992; Scott 1992). Comparative studies of mammalian homeo box gene clusters with the Drosophila homeotic complex $(H O M-C)$ have shown numerous similarities in protein sequences, gene organization, and patterns of expression suggesting that the organization of these complexes predates the evolutionary divergence of insects and verte-

${ }^{6}$ Corresponding author. brates (Schughart et al. 1989). This evolutionary conservation also raises the issue as to whether the functions of these proteins have been similarly conserved.

Possible developmental functions for the mammalian Hox genes have been deduced, in part, from circumstantial evidence. For example, all Hox genes examined display distinct temporally and spatially regulated patterns of expression in the developing embryo. Typically, expression is initiated during gastrulation in the mesoderm and ectoderm lineages and continues up to mid-gestation and early organogenesis (Holland and Hogan 1988). The majority of Hox genes have overlapping domains of expression in the neural tube and prevertebrae that extend from the caudal end of the embryo to a defined anterior limit. The anterior boundaries of expression appear to correlate (with few exceptions) to the linear order of the genes within a given cluster. Consequently, different members of the Hox clusters are expressed in discrete but overlapping regions along the anteroposterior axis of the embryo. It has been proposed from these observations that the specific combination of different Hox genes expressed in a particular axial region provides a unique genetic address specifying the characteristics, or identity, of a particular axial level (Kessel and Gruss 1991). Consistent with this hypothesis, grafting individ- 
ual somites that express the gene Hoxa-7 (Hox1.1) to heterotopic positions in the anteroposterior axis of mouse embryo is not sufficient to respecify $\mathrm{Hoxa}-7$ gene expression (Beddington et al. 1992; Gruss and Kessel 1991).

Genetic approaches have been used to directly test the function of Hox genes in governing vertebral identity in the intact embryo. A number of dominant gain-of-function mutations have been reported in transgenic mice. The outcome of these experiments depends on the gene studied and the promoter elements used. Some transgenic strains show homeotic posteriorization analogous to that described in Drosophila following ectopic HOM-C gene expression (Balling et al. 1989; Lufkin et al. 1992; McLain et al. 1992). In contrast, other transgenes produced anterior transformations, a result more in keeping with phenotypes observed for loss of function of Drosophila homeotic genes (Jegalian and De Robertis 1992; Pollock et al. 1992). In cases leading to posteriorizing transformation, the morphological alterations appear to be limited to embryonic regions lying anterior to the normal domain of gene expression. One interpretation of these experiments is that ectopic expression of specific Hox genes in more anterior regions of the embryo is sufficient to cause the repatterning of anterior skeletal structures (Balling et al. 1989; Lufkin et al. 1992 ). In the case of transgenes that give anterior transformations, vertebral changes arise in the posterior regions of the embryo where the genes are normally expressed, suggesting that the relative quantitative level of expression of particular Hox genes has an important role in specifying vertebral identity (Jegalian and De Robertis 1992; Pollock et al. 1992). Thus, in both of these experimental situations, perturbing the pattern of expression of single Hox genes clearly has important consequences on the development of the embryo.

An alternative approach is to eliminate Hox gene function. Gene targeting in embryonic stem (ES) cells has allowed the production of strains of mice carrying predetermined mutations in a number of Hox gene loci (Chisaka and Capecchi 1991; Lufkin et al. 1991; Chisaka et al. 1992; LeMouellic et al. 1992; Ramirez-Solis et al. 1993). Loss of Hoxa-3 (Hox1.5) and Hoxa-1 (Hox1.6) function results in morphological defects largely confined to the more anterior domains of normal gene expression and, interestingly, does not influence the formation of axial structures (Chisaka and Capecchi 1991; Lufkin et al. 1991; Chisaka et al. 1992). In both mutants, the malformations appear to be structural deficiencies resulting from perturbations to neural crest populations rather than homeotic transformations and are therefore more analogous to phenotypes obtained from loss of function of the labial gene, the Drosophila homolog of Hoxa-1 (Merrill et al. 1989). In contrast, mutation of the Hoxc-8 (Hox3.1) and Hoxb.4 (Hox2.6) genes that have more posterior boundaries of expression results in anterior transformation of skeletal segments that normally express these genes (LeMouellic et al. 1992; RamirezSolis et al. 1993). These abnormalities thus resemble those observed for some $H O M-C$ mutations [such as
Antp, Ultrabithorax (Ubx), abdominal-A (abd-A), and $A b d o m i n a l-B(A b d-B \mid]$ and suggest that subsets of $H o x$ genes may contribute in determining the identity of axial structures. Intriguingly, the vertebral transformations observed in the Hoxc- 8 mutant mice appear to be similar to those obtained in transgenic animals overexpressing either Hoxc-8 or Hoxc-6 (Hox3.3). Collectively, these data argue that the precise quantitative contribution by specific Hox genes plays an important role in controlling vertebral identity (Jegalian and De Robertis 1992; Pollock et al. 1992).

We have been studying the Hoxa-5 gene which, in common with other mammalian Hox genes, is expressed in a distinct domain along the anteroposterior axis of the developing embryo. In the 8-day-old embryo, Hoxa-5 expression is restricted to the posterior presomitic mesoderm (Dony and Gruss 1987). One day later, Hoxa-5 transcripts are detected primarily in the somites of the thoracic region, suggesting that Hoxa-5 expression is confined to those cell populations that ultimately give rise to this region of the body (Dony and Gruss 1987). At 12.5 days of gestation, Hoxa-5 transcripts are present in the prevertebral column, with expression extending from pv24 (which corresponds to the fourth lumbar vertebra) to a rostral boundary that coincides with pv3 (the third cervical vertebra) (Gaunt et al. 1988). The greatest abundance of transcripts is found in the region encompassing pv6 to pv14 (Gaunt et al. 1988, 1990). The gene is also expressed in the ribs and in other mesodermal derivatives such as metanephros, lung, stomach, and intestine (Dony and Gruss 1987; Gaunt et al. 1988). Within the developing spinal cord, the anterior limit of Hoxa-5 expression coincides with the posterior floor of the myelencephalon (Gaunt et al. 1988). On the basis of protein sequence comparisons, Hoxa-5 can be grouped in the same subfamily as Hoxb-5 (Hox2.1) and Hoxc-5 (Hox3.4). These three genes have been shown to have similar domains of expression along the anteroposterior axis (Gaunt et al. 1990).

The Hoxa-5 gene encodes a nuclear phosphoprotein capable of binding to specific DNA sequences. The presence of Hoxa-5-binding sites has been reported in the putative Hoxa-5 regulatory regions and in cis-regulatory elements of other Hox genes, transcription factor genes, and viral genes (Odenwald et al. 1989). Thus, Hoxa-5 protein may act in both an auto- and a cross-regulatory fashion, similar to that described for a number of Drosophila loci (Hayashi and Scott 1990).

To examine whether the Hoxa-5 gene has an essential developmental function and, more specifically, to determine any contribution by this homeo domain protein in the establishment of axial identity, we have used gene targeting in ES cells to generate a line of mice that carry a disrupted Hoxa-5 gene (Jeannotte et al. 1991). Although mice that are heterozygous for the mutation are phenotypically normal, roughly half of those animals homozygous for the disrupted Hoxa-5 allele die at birth or shortly thereafter. Analysis of the skeletal structures of homozygotes revealed consistent abnormalities affecting the region between the sixth cervical vertebra $(\mathrm{C} 6)$ and 
the first lumbar vertebra (L1). The most frequent morphological changes are the absence of tuberculi anterior on $\mathrm{C} 6$ and the presence of cervical ribs on the seventh cervical vertebra (C7) causing it to assume an appearance typical of a thoracic vertebra. Taken together, these results show that Hoxa-5 plays a central role in specification of axial identity in the developing embryo.

\section{Results}

\section{Inactivation of the Hoxa-5 gene in mice}

We previously described the generation of ES cell lines in which the DNA-binding homeo domain of the Hoxa-5 gene is interrupted by the introduction of a neo gene. (Jeannotte et al. 1991). Targeted clones were obtained by using either a promoterless gene targeting vector or by a positive-negative selection procedure. In both cases, the mutation generated will, at best, result in the production of a truncated Hoxa-5 molecule lacking the final 58amino-acid residues of the protein. This region contains the third helix of the homeo domain thought to confer DNA-binding specificity of Hox proteins /Otting et al. 1990; Gehring 1992). A correctly targeted ES cell clone (designated M-31-13), generated with the positive-negative targeting vector, gave germ-line chimeric mice. The resultant heterozygous Hoxa-5 mutant mice were viable and indistinguishable from their wild-type littermates, and heterozygotes of both sexes proved fertile. Hoxa-5 heterozygous $F_{1}$ animals were mated with MF1 strain partners to expand the pool of mice carrying the mutant allele.

\section{Hoxa-5 inactivation is associated with a high rate of perinatal lethality}

To assess the consequence of loss of Hoxa-5 function, heterozygous Hoxa-5 mice were intercrossed. The resulting offspring were genotyped at 3-4 weeks of age by

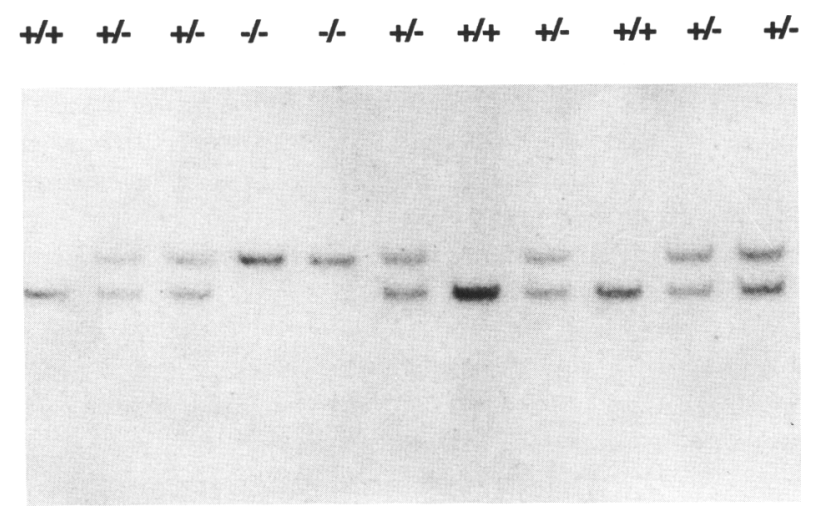

Figure 1. Southern analysis of offspring obtained from heterozygous crosses. Tail DNAs from 3- to 4-week-old pups derived from Hoxa-5 m/ + intercrosses were digested with EcoRV, blotted, and probed with a Hoxa-5 exon 2 BglII-HindIII probe. The genotype of each animal is indicated at the top of each lane. $(+)$ Wild-type allele, lower band; $(-)$ disrupted Hoxa-5 allele, upper band.
Table 1. Ratios of genotypes in litters from crosses of heterozygous Hoxa-5 mutants

\begin{tabular}{lccccc}
\hline & & & \multicolumn{3}{c}{ Genotype } \\
\cline { 5 - 6 } Age & $\begin{array}{c}\text { Number } \\
\text { of litters }\end{array}$ & $\begin{array}{c}\text { Number } \\
\text { of pups }\end{array}$ & $\begin{array}{c}+/+ \\
(\%)\end{array}$ & $\begin{array}{r}+/- \\
(\%)\end{array}$ & $\begin{array}{r}-/- \\
(\%)\end{array}$ \\
\hline $\begin{array}{c}12.5 \text { days } \\
\text { p.c. }\end{array}$ & 18 & 170 & $37(22)$ & $104(61)$ & $29(17)$ \\
$\begin{array}{c}18.5 \text { days } \\
\text { p.c. }\end{array}$ & 11 & 104 & $27(26)$ & $47(45)$ & $30(29)$ \\
$3-4$ weeks & 85 & 619 & $195(31)$ & $344(56)$ & $80(13)$ \\
\hline
\end{tabular}

Southern blot analysis of genomic DNA digested with EcoRV (Fig. 1). Under these conditions, the wild-type gene is visualized as a $7-\mathrm{kb}$ band and the mutant allele as an 8 -kb band. From a total of 619 progeny analyzed, only 80 animals homozygous for the Hoxa-5 mutation were obtained. This corresponds to some $13 \%$ of offspring instead of the expected $25 \%$, assuming the mutation was inherited in a Mendelian fashion (Table 1). To determine at what stage the mutant animals were dying, heterozygous Hoxa-5 animals were intercrossed and pregnant females were sacrificed at different times in gestation [the day at which the vaginal plug was found was designated 0.5 days postcoitum (p.c.)]. The embryos were dissected, and individual visceral yolk sac tissues were used as a source of genomic DNA for genotyping. As shown in Table 1, at 18.5 days p.c., the numbers of wild-type embryos, heterozygotes, and homozygotes were found to conform to the expected $1: 2: 1$ ratio. No overtly abnormal embryos were observed. Curiously, at 12.5 days p.c., the relative proportion of the progeny deviates from the expected ratio of $1: 2: 1(P<0.01)$. This difference is difficult to explain because the expected ratios were recorded at 18.5 days p.c.. The absence of Hoxa-5 function thus appears to lead to significant mortality between birth and weaning.

The fraction of homozygous mutant animals that survived to adulthood proved to be fertile and did not display any obvious phenotypic abnormalities. Matings were set up between heterozygous and homozygous animals (Table 2). At 18.5 days p.c., of 69 embryos retrieved for analysis, half proved to be homozygotes. Careful monitoring of litters during the first week of postnatal life showed that $61 \%$ of the homozygous pups died within 4 days of birth. Finally, homozygous animals were intercrossed. The survival rate of the resultant homozygous pups after 1 week was only $47 \%$ (of 99 mice born; data not shown), and the majority of pups died at birth or shortly thereafter. In general, homozygous pups that died during this time period appeared normal on the day of birth and were able to suckle, but subsequently displayed a general failure to thrive. Gross necropsy of selected animals did not reveal any overt tissue and organ defects that could account for their death.

\section{Expression of wild-type and mutant Hoxa-5 alleles}

Restriction analyses of the targeted Hoxa-5 allele present 
Table 2. Ratios of genotypes in litters from crosses of heterozygous Hoxa-5 mutants with homozygous Hoxa-5 mutants

\begin{tabular}{|c|c|c|c|c|c|c|}
\hline \multirow[b]{3}{*}{ Age } & \multirow[b]{3}{*}{ Number of litters } & \multirow[b]{3}{*}{ Number of pups } & \multicolumn{4}{|c|}{ Genotype } \\
\hline & & & \multicolumn{2}{|c|}{$+1-$} & \multicolumn{2}{|c|}{$-1-$} \\
\hline & & & living $(\%)$ & $\operatorname{dead}^{a}\langle \%\rangle$ & living $(\%)$ & $\operatorname{dead}^{\mathrm{b}}(\%)$ \\
\hline 18.5 days p.c. & 7 & 69 & $37(54)$ & N.A. ${ }^{c}$ & $32(46)$ & N.A. ${ }^{c}$ \\
\hline Birth & 14 & 126 & $60(48)$ & $4(3)$ & $24(19)$ & $38(30)$ \\
\hline
\end{tabular}

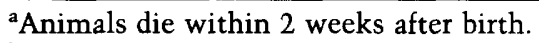

bAnimals die at birth or within 4 days after birth.

'(N.A.) Data not available.

in clone M-31-13 confirmed that the intended replacement mutation had occurred (Jeannotte et al. 1991). To confirm that this mutation had affected the synthesis of Hoxa-5 transcripts, Northern blot analysis was performed. RNA isolated from lung, kidney, and intestine of wild-type, heterozygous, and homozygous adult animals was assayed by hybridization with a Hoxa-5 exon 2 probe. This probe derives from a region $3^{\prime}$ of the homeo domain and detects four transcripts of approximately 2 , 4,8 , and $9 \mathrm{~kb}$ in length in RNA samples from 12.5 day embryos and some adult tissues (Fig. 2; Odenwald et al. 1987). In contrast, in samples from homozygous mutant animals, the RNA species corresponding in size to the wild-type transcripts were absent, and only transcripts 1 $\mathrm{kb}$ longer than the wild-type Hoxa-5 transcripts were present (Fig. 2). As expected, these novel transcripts

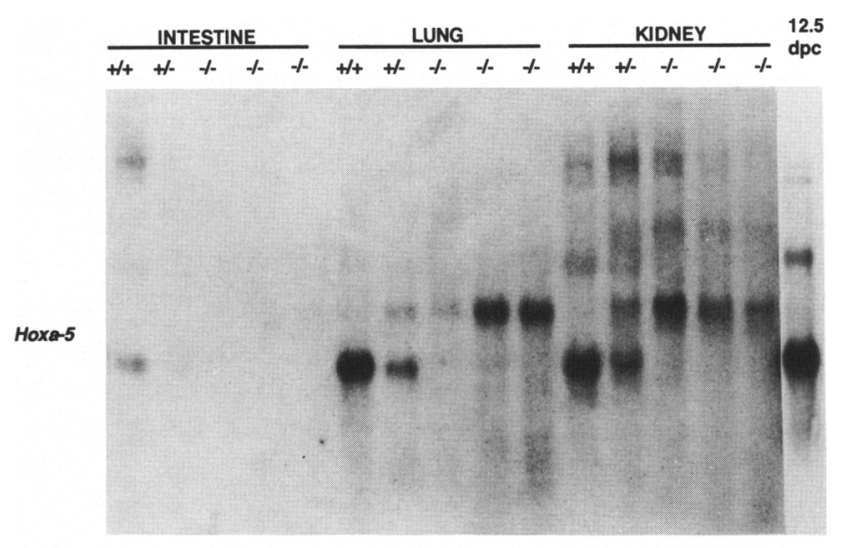

$\alpha-$ actin

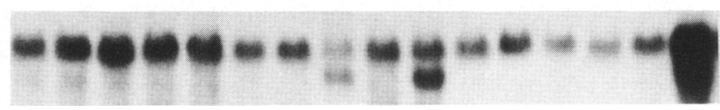

Figure 2. Expression of the Hoxa-5 gene in Hoxa-5 wild-type, heterozygous, and homozygous mutant animals. Total RNA 120 $\mu \mathrm{g}$ ) was isolated from adult intestine, lung, and kidney tissues, fractionated by electrophoresis through a formaldehyde-agarose gel, transferred to Hybond membrane, and hybridized to a ${ }^{32} \mathrm{P}$. labeled Hoxa-5 exon 2 BgllI-HindIII probe. One wild-type $1+1$ +1 , one heterozygous $(+1-1$, and three individual homozygous mutants $(-/-)$ were analyzed. Poly $(\mathrm{A})^{+}$RNA $(5 \mu \mathrm{g})$ from normal 12.5-day-old embryos was used as a control to illustrate the sizes of endogenous Hoxa-5 transcripts. To demonstrate RNA integrity, the blot was rehybridized to a mouse $\alpha$-actin probe. proved to contain neo sequences (data not shown), suggesting that they derived from the mutated allele with the size increase being attributable to the insertion, in the opposite transcriptional orientation, of the $1-\mathrm{kb}$ $\mathrm{MClneo} \mathrm{A}^{+}$cassette within the homeo box sequence (as described in Jeannotte et al. 1991). Thus, integration of the targeting vector eliminated expression of normal Hoxa-5 mRNAs. In heterozygotes, both the wild-type and mutated forms of Hoxa-5 transcripts were present, although expression of transcripts derived from the mutated allele appeared to be significantly reduced. This presumably resulted either from a reduction in the stability of these transcripts owing to the presence of the neo sequences or reduced transcriptional activity of the targeted allele.

\section{Hoxa-5 mutants show homeotic transformations of the skeleton}

To determine whether mutation of the Hoxa-5 gene affected the formation of structures derived from tissues in which the gene is normally expressed, we examined the skeletons of perinatal animals (between 18.5 day p.c. embryos and birth). The majority of homozygous animals displayed a variety of skeletal abnormalities restricted to the cervical and thoracic regions (Table 3). The most predominant of these was a phenotypic alteration of $\mathrm{C} 7$ which, in many animals, was found to have associated rib structures. Indeed, $73 \%$ of the homozygous mice examined had extensive ribs on one or both sides of $\mathrm{C7}$ (Figs. 3 and 4). In approximately one-third of cases, these ribs were fused ventrally with the ribs associated with the first thoracic vertebra (Fig. 3C). In some cases, cervical ribs made an independent contact to the sternum (Figs. 3D and 4A). On occasion, extra ribs were seen in wild-type animals, but in these cases they took the form of small anlages (Table 3). In heterozygous embryos, the number of instances of small cervical ribs associated with $\mathrm{C7}$ was markedly increased, but only rarely were animals found that possessed an extensive rib. This may indicate that the specification of this vertebra could be particularly sensitive to Hoxa-5 gene dosage effects. Analysis of adult homozygotes revealed the same morphological change of $\mathrm{C7}$, but the proportion of animals with pronounced long cervical ribs was reduced (only 3 of 21 animals analyzed; data not shown). Thus, the mu- 
Table 3. Skeleton morphology according to Hoxa-5 genotypes

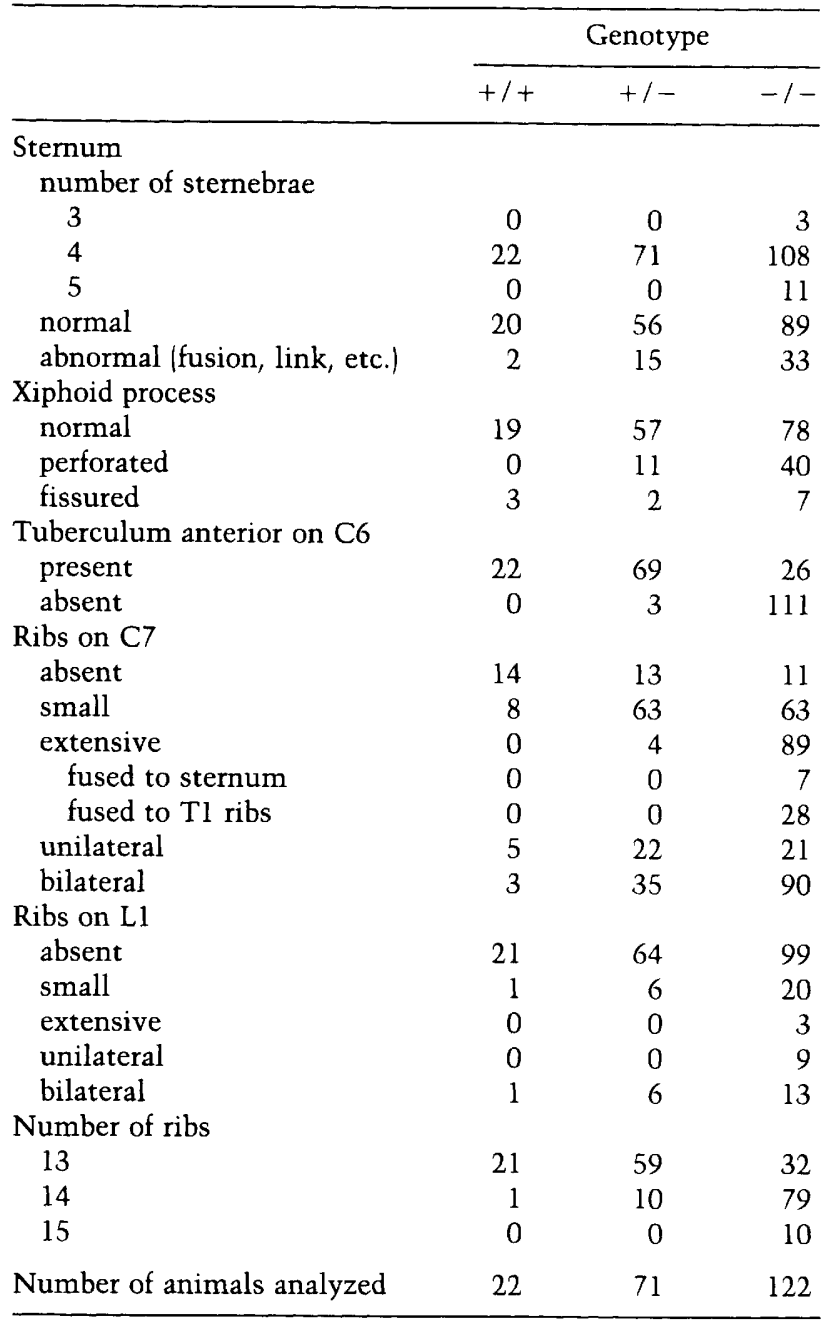

tant animals that display a strong $\mathrm{C} 7$ phenotype represent a significant proportion of the class of homozygotes that die during the perinatal period.

A second frequent phenotypic change seen in the Hoxa-5 homozygous mice was the absence of tuberculum anterior on C6, which occurred in more than $90 \%$ of the embryos and adult animals (Figs. 3 and 4). This was seen most easily in adults, following complete ossification of the tuberculum anterior (Fig. 4B). In transverse view, C6 becomes morphologically very similar to C3, $\mathrm{C} 4$, and $\mathrm{C} 5$, and this alteration does not interfere with the entry of the vertebral artery into the C6.

The phenotypic transformation of $\mathrm{C} 7$ into a thoracic vertebra confers a different vertebral distribution (C6/ T14/L6) in the majority of the homozygotes. Additionally, the presence of ribs on the first lumbar vertebra was noted in 19\% of homozygous embryos (Table 3; Fig. 5). This second transformation event, in combination with that affecting $\mathrm{C}$, leads to the generation of a small proportion of mutants $(8 \%)$ that possessed 15 as opposed to 13 pairs of ribs. However, it is important to note that while the identity of the vertebrae is altered in the Hoxa-5 mutants, no cases were found in which the total number of presacral vertebrae was altered in comparison to control animals, indicating that the changes documented in mutant animals are homeotic.

A small proportion of homozygous animals were found to have defects of the sternum (Fig. 6). In wild-type and heterozygous animals, in addition to the manubrium sterni and the xiphoid process, 4 sternebrae were present in the intercostal region. The attachment sites of the first six ribs are normally separated by sternebrae, whereas the sixth and seventh ribs attach to the sternum at the same point. In $9 \%$ of the homozygotes examined, an extra sternebrae was present that appeared to form by fission of two of the manubrium sterni. This is illustrated in the animal shown in Figure 6B, in which the first ribs derived from $\mathrm{C} 7$ and were fused to the sternum at a position normally occupied by the first pair of thoracic ribs. A consequence of this displacement is that the first thoracic ribs joined the sternum in the center of the manubrium sterni, which appeared to be divided in these animals. In addition, the sternebra anterior to the xiphoid process (which normally corresponds to the fourth sternebra| was reduced in size and linked to the preceeding sternebra. Overall, this resulted in a close juxtapositioning of the sites of attachment of the fifth, sixth, and seventh ribs. In a further three homozygous animals, fusion of adjacent sternebrae was observed resulting in a decrease in their number and production of irregularities in the sternocostal junctions. Figure 6C shows an example of a sternum in which the attachment of the ribs was asymmetric. In addition, ossification of the xiphoid process was altered in many mutants (Fig. 6B,C). The xiphisternum normally develops from two centers of ossification, which initially lie to either side of the ventral midline and which rapidly fuse together across the midline (Kaufman 1992). Thus, at 18.5 days p.c., a discrete hole is sometimes apparent in the central part of the xiphoid cartilage. However, in the case of the homozygous mutants animals, the frequency of holes and fissures was significantly higher than that found in wild-type and heterozygous animals $(39 \%$ vs. $14 \%$ and $19 \%$, respectively) and the malformations were not necessarily centrally located along the midline.

Analysis of a large sample of mutants showed that all of the skeletal transformations described above were often, but not always, found in the same animal. For example, it was noticeable that animals showing either anterior transformation of the first lumbar vertebra or the sixth cervical vertebra also consistently showed posterior transformation of the seventh cervical vertebra. Although these transformations varied in their physical extent between individual mutants, the penetrance of the transformation was highest in $\mathrm{C} 6$ and $\mathrm{C} 7$ and lowest between $\mathrm{Tl}$ and $\mathrm{L} 1$. Thus, the region encompassing $\mathrm{C} 6$ to $\mathrm{L} 1$ seemed to delineate the extent of skeletal transformations that occur in Hoxa-5 mutant mice. The basis of the variable expressivity of the skeletal phenotypes that characterize Hoxa-5 mutants is not known; but as the animals used in the current study were maintained in a 
Figure 3. Skeletal transformations affecting the cervicothoracic region in Hoxa-5 mutants. Skeletons of animals ranging in age from 18.5 day p.c. embryos to newborn animals were stained with alizarin red (visualizes the bones) and alcian blue (visualizes the cartilage). (A) Lateral view of a wild-type embryo showing the cervicothoracic transition region. Note the absence of ribs on the seventh cervical vertebra and the presence of tuberculum anterior on the sixth cervical vertebra. $(B-D)$ Lateral view of Hoxa-5 homozygous mutant embryos showing a rib on the seventh cervical vertebra. In $C$, the cervical rib appears to be fused ventrally with the first thoracic rib, while in $D$, cervical ribs make an independant contact to the sternum. In the three homozygous mutant embryos, the C6 vertebrae lack a tuberculum anterior. The arrowheads indicate the cervical ribs.
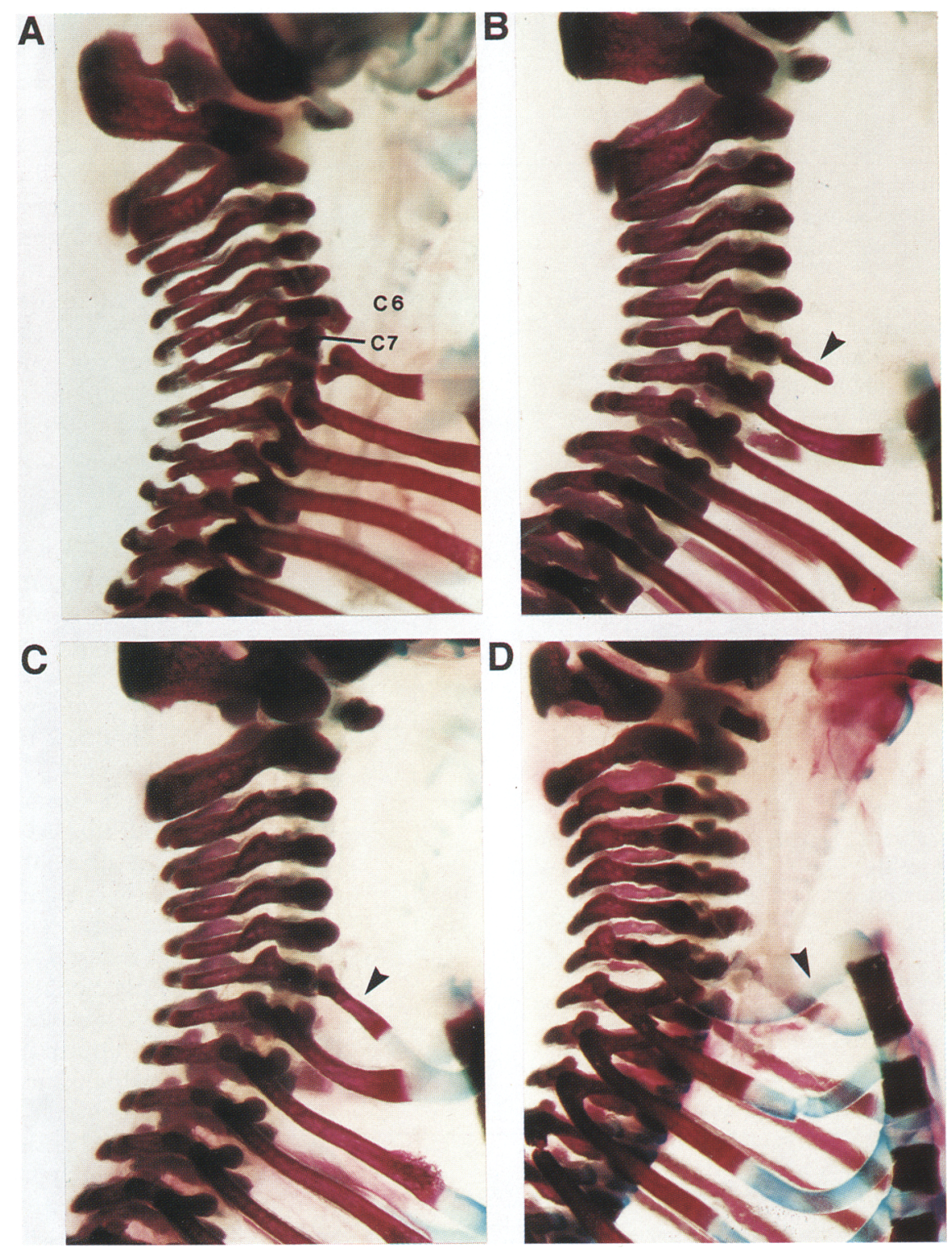

hybrid genetic background, subtle genetic differences may be a contributory factor, as has been documented recently for the Hoxb-4 mutation (Ramirez-Solis et al. 1993).

\section{Discussion}

We have used gene targeting to generate a mutation at the Hoxa-5 locus. In this mutation, the introduction of the neo gene into the homeo domain of the Hoxa-5 locus is predicted to generate a loss-of-function allele. Our analysis of the resultant mutant mouse strain has shown that loss of function of the Hoxa-5 gene results in a dramatic decrease in viability of homozygotes at birth. Moreover, the mutation leads to homeotic transformations of the vertebral column in homozygous mutants.

Expression studies are highly suggestive that mouse
Hox genes act in concert to specify positional information along the anteroposterior axis of the developing embryo in a manner analogous to their Drosophila counterparts. Mutations in Drosophila homeotic genes result in abnormal development of specific segments of the embryo (Akam 1987). According to the model proposed by Lewis (1978), loss-of-function mutations should transform segments into the likeness of more anterior ones while gain-of-function mutations are predicted to give transformations to more posterior segments. However, it is obvious that this model does not hold true because ectopic expression of either Deformed $(D f d)$ or Sex combs reduced $(S \mathrm{Cr})$ confers a more anterior identity to posterior epidermal cells (Wakimoto and Kaufman 1981; Merrill et al. 1987; Kuziora and McGinnis 1988). Similarly, lack of Scr function leads to a partial transformation of the labial segment to a maxillary segment and of 

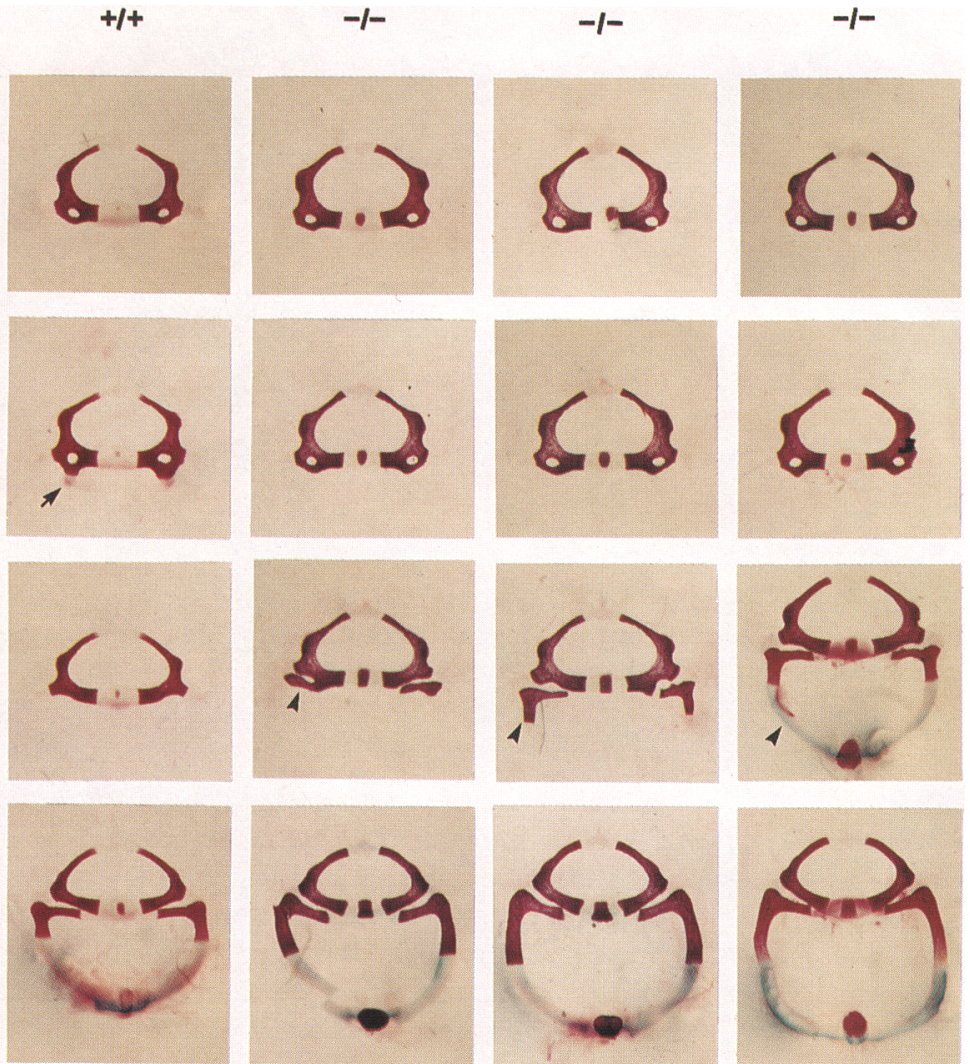
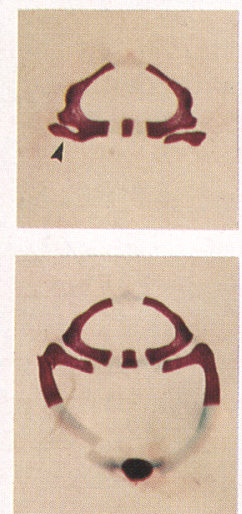
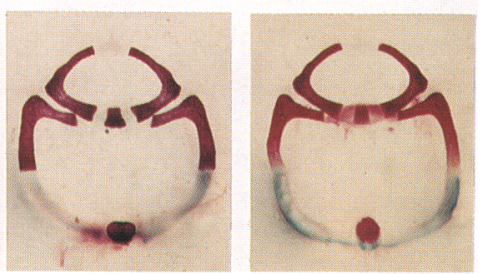

B

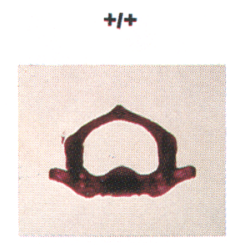

C4

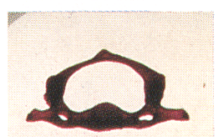

C5

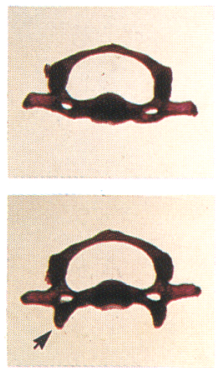

C7

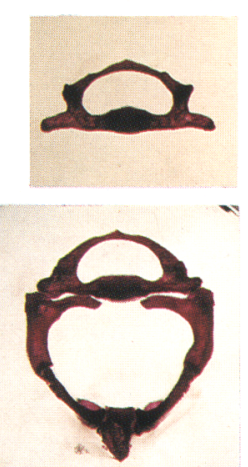

$-1-$
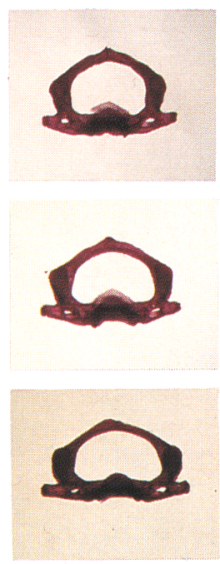

c6

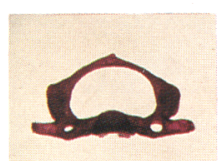

T1
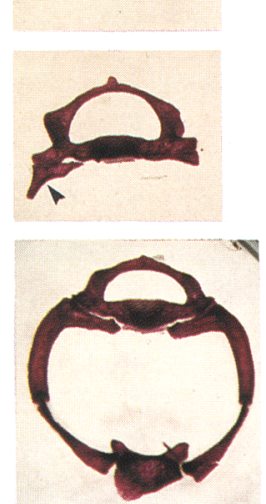

Figure 4. Transformation of cervical vertebrae in Hoxa-5 mutants. Vertebrae were dissected from clearing preparations by cutting the intervertebral disc and the sternum from wild-type $|+|+\mid$ and mutant $|-|-\mid 18.5$-day-old embryos $(A \mid$ and adults $(B \mid$. In homozygous animals, the tuberculum anterior (completely ossified in adults) is absent on the sixth cervical vertebra. The morphological appearance of $\mathrm{C} 6$ then becomes strikingly similar to $\mathrm{C} 3, \mathrm{C} 4$, and $\mathrm{C} 5$. Homozygous animals had ribs on the seventh cervical vertebra in some cases, which then closely resembles the first thoracic vertebra $(A)$. The arrows indicate the tuberculum anterior; the arrowheads show the cervical ribs.

the prothoracic segment to the mesothoracic segment in the embryo, both of which represent posterior transformations (Wakimoto and Kaufman 1981). Complete elimination of $S c r$ gene function reveals the function of the overlapping, more posterior Antp gene (Kaufman et al. 1990). In Drosophila, homeotic transformations that arise from loss-of-function mutations are only observed for those genes having expression domains that overlap those of other HOM-C genes. Thus, Scr, which belongs to the group of HOM-C genes that correspond the Drosophila homologs of Hoxa-5, differs from other homeotic genes in not conforming to the model proposed by Lewis (1978).

We have documented both categories of morphological transformations in Hoxa-5 homozygous mutant mice. Anterior transformations were seen to affect $\mathrm{C} 6$, causing it to adopt a morphological appearance strikingly similar to the more anterior vertebra C5. Additionally, in a smaller number of cases, the first lumbar vertebra was transformed into a thoracic vertebra based on the appearance of ribs. However, a very common observation in mutant animals was that $\mathrm{C} 7$ adopts a more posterior fate and is transformed into a thoracic vertebra. As mutations in $\mathrm{Scr}$ do not give the expected transformations, together with our results showing that Hoxa-5 mutants display a posterior transformation, these data reinforce the hypothesis that both genes are members of the same subfamily. On the basis of sequence homology within the homeo domain, relative position within their respective gene clusters, and the type of transformations that accompany their mutation, a strong argumant can be made that Scr is the true Drosophila homolog of Hoxa-5. Moreover, it was shown recently that ectopic expression of mouse Hoxa-5 sequences in Drosophila results in Scrlike transformations and that Hoxa-5 can participate in Drosophila regulatory networks by activating down- 

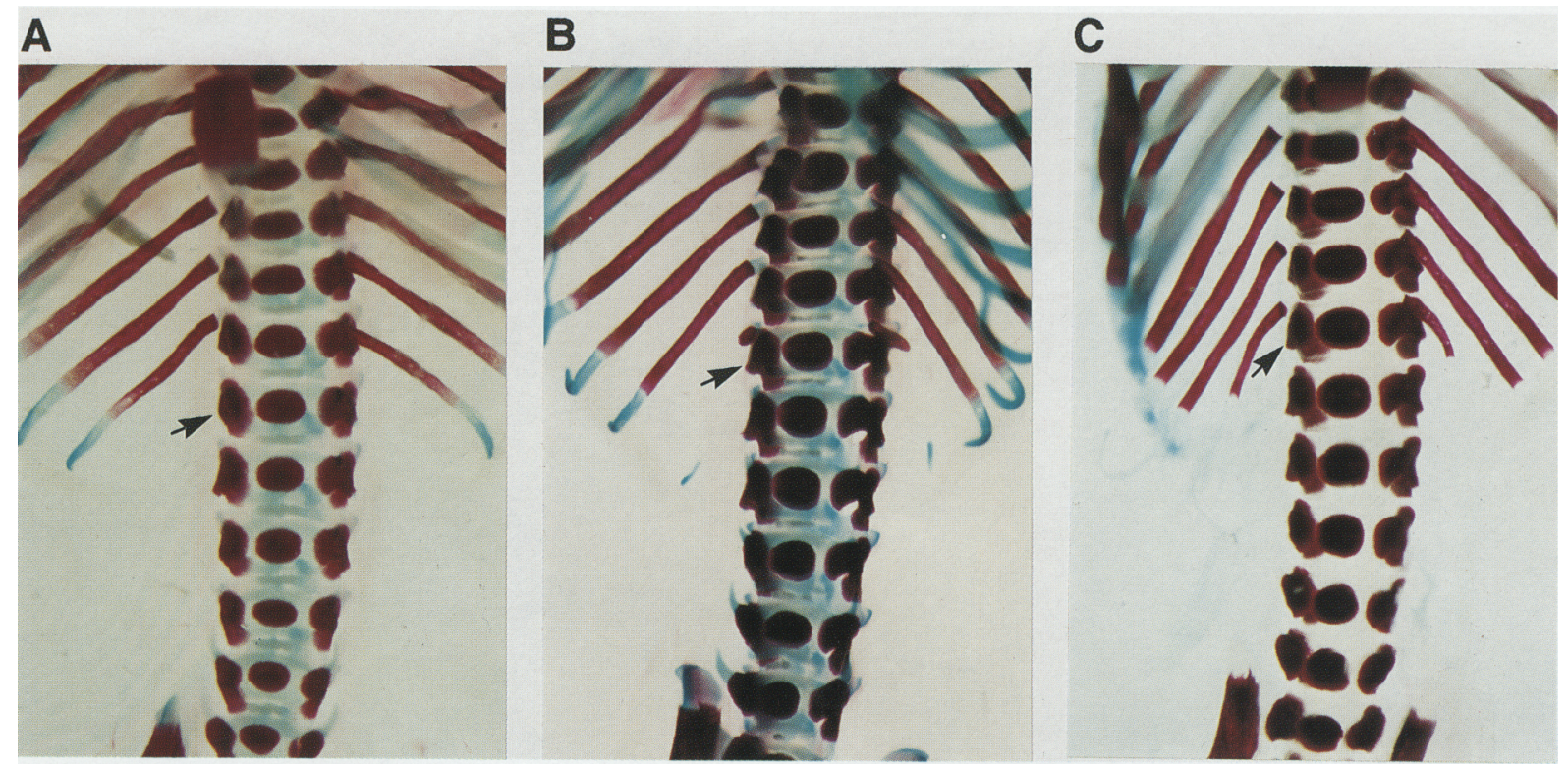

Figure 5. Transformation of the first lumbar vertebra in Hoxa-5 mutants. (A) Ventral view of a wild-type 18.5 day p.c. embryo showing the absence of ribs on the first lumbar vertebra. $(B, C)$ Ventral view of Hoxa-5 homozygous mutant embryos showing a pair of short ribs on the first lumbar vertebra $(B)$ and more extensive ribs $|C|$. The arrows indicate the first lumbar vertebra.

stream targets of Scr (Zhao et al. 1993). Collectively, these results strongly suggest that Hoxa-5 and $S c r$ are cognate genes. It will be interesting to analyze the phenotypic consequences of introducing mutations into the two mouse Hoxa-5 paralogs.

In Drosophila, mutational studies have indicated that important functional roles of specific $H O M-C$ genes seem to be confined to the subregions that accumulate the highest levels of expression within a more extensive expression domain (McGinnis and Krumlauf 1992). Generally the highest levels of mammalian Hox gene expression are found in tissues corresponding to the more anterior regions of expression. If quantitative differences in the absolute levels of gene expression are important functionally, it might be expected that these regions of the embryo would show the most severe perturbations (Hunt and Krumlauf 1992). In Hoxa-5 mutant mice, the most frequent morphological changes are confined to a small region of the axis that includes the sixth and seventh cervical vertebrae. The highest level of Hoxa-5 expression, as assessed by in situ hybridization, is in the region including pv6 to pvl4 (C6 and T7, respectively), with lower levels of gene expression extending more caudally (Gaunt et al. 1988). The pv6-pv14 region includes the region of the thorax that participates in the formation of the sternum. Because Hoxa-5 is expressed in the mesodermal condensation that gives rise to the sternum and ribs precursors, abnormalities at the level of the sternum can be attributed to the absence of functional Hoxa-5 gene in this region of the embryo. In Hoxa-5 mutant mice, the affected region of the body axis is thus entirely contained within the normal expression domain of the gene.

The posterior prevalence model does not adequately explain the mode of action of the Hoxa-5 mutation, as transformations of identity occur in axial regions that express Hox genes located $5^{\prime}$ to the Hoxa-5 gene in the cluster (namely genes that have a more posterior domain of expression). The basis of this model is that positional specification is largely achieved by the dominant action of the most posterior homeotic gene at a given axial level. Lack of one component should theoretically result in a group of several vertebrae at a specific axial level adopting a single fate, rather than a more extended series of vertebrae adopting the morphology of immediately adjacent ones (Hunt and Krumlauf 1992). The Hoxa-5 mutant phenotype is thus better explained by models in which quantitative levels of expression have a critical role. Similar findings have been described in animals mutant for the Hoxc-8 gene, which is also expressed in the trunk region (LeMouellic et al. 1992; Pollock et al. 1992). Thus, loss of function of either of these two genes results in abnormalities that span a more extensive region than that documented in mice mutant for the Hoxa-1 and Hoxa-3 genes expressed more anteriorly, which show defects confined to the branchial region (Chisaka and Capecchi 1991; Lufkin et al. 1991; Chisaka et al. 1992).

Presence of cervical ribs on $\mathrm{C} 7$ has been recorded in embryos exposed to teratogenic doses of retinoic acid, whereas anterior transformation of C6 into C5 was reported in RAR $\gamma$ homozygous mutants (Kessel and Gruss 1991; Kessel 1992; Lohnes et al. 1993). Retinoic acid is known to modulate expression of Hox genes in both cultured cells and in vivo (Kessel and Gruss 1991; Papalopulu et al. 1991; Simeone et al. 1991; Kessel 1992). In embryos treated in utero with retinoic acid, an overall anterior extension of the normal domains of expression 
A

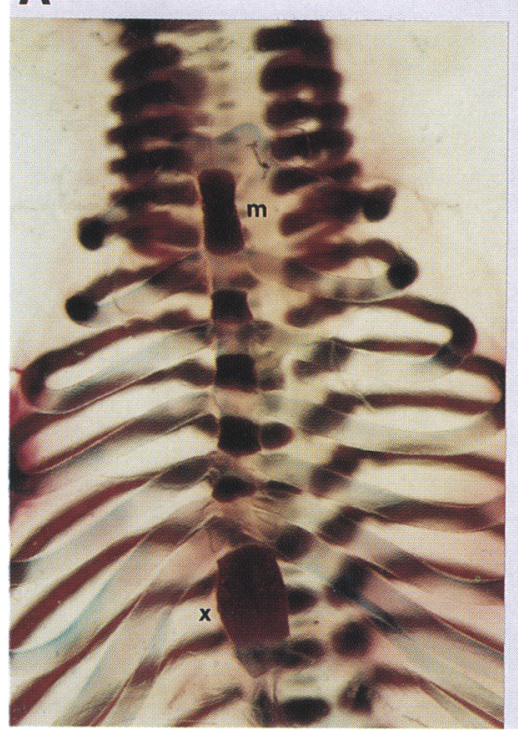

B

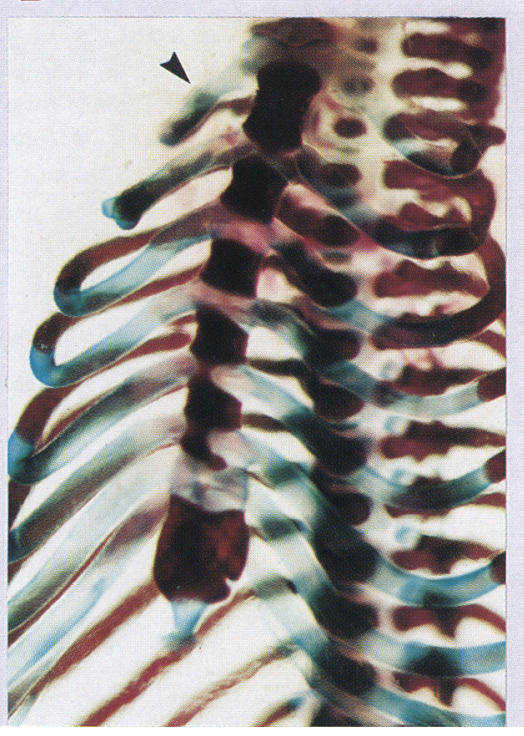

C

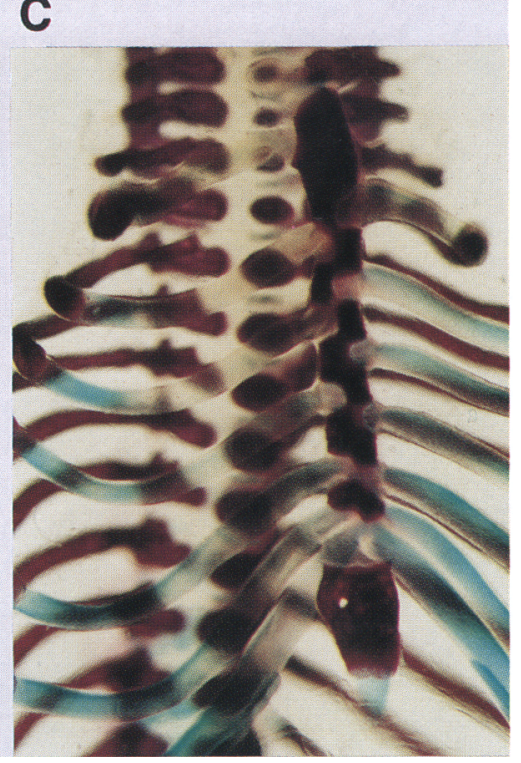

Figure 6. Hoxa-5 mutants show abnormalities of the sternum. $(A)$ Ventral view of a wild-type 18.5 day p.c. embryo showing the sternum region. Normally, seven pairs of ribs are attached to the sternum, which contains four sternebrae in addition to the manubrium sterni and the xiphoid process. $(B, C)$ Ventral views of the sternum region of mutant embryos. In $B$, the cervical ribs are fused to the sternum at the top of the manubrium sterni at the position normally occupied by the first thoracic ribs. The first thoracic ribs join the sternum in the middle of the manubrium sterni, which as a consequence appears to be split in two. The last two sternebrae before the xiphoid process are linked together, resulting in a close juxtaposition of the sites of attachment of the fifth, sixth, and seventh thoracic ribs. In $C$, fusion of adjacent sternebrae results in irregularities in the sternocostal junctions. Ossification of the xiphoid process also appears altered, as fissures and holes are observed $(B, C)$. (m) Manubrium sterni; $(\mathbf{x})$ xiphoid process; $($ arrowhead) cervical rib.

of specific Hox genes was reported that correlate with the induced alterations in morphology (Kessel and Gruss 1991; Kessel 1992; Marshall et al. 1992). One caveat is that it is not yet clear whether the morphological effects can be attributed solely to alterations in Hox gene expression.

In addition to expression in the spinal cord, Hoxa-5 mRNA is localized to mesoderm structures derived from the somites, the lateral mesoderm, and the lateral plate. Gross analysis of homozygous pups did not reveal any major perturbations of tissues or organs that normally express the Hoxa-5 gene. However, clearly the major phenotype presented by Hoxa -5 mutant mice is a greatly reduced viability during the first few days of postnatal life. We note that approximately half of the mutant animals die within the first week of birth and that the reduction in viability is intrinsic to the mutant animals as the loss of pups occurs irrespective of the genotype of the mother. Thus, loss of Hoxa-5 function in some way interferes with the correct physiological functioning of major organ systems. The Hoxc-8 mutation also results in very marked postnatal lethality $(\sim 6 \%$ of mutants survived to adulthood), with the surviving animals showing neurological disorders and slight runting (LeMouellic et al. 1992). In contrast, the surviving Hoxa-5 mutants appear indistinguishable from wild-type or heterozygous siblings in behavior, reproductive performance, and longevity. The variability in expressivity of the mutation may result from segregation of "modifier" loci. This interpretation is reinforced by recent data showing that one of the Hoxb-4 mutations results in high rates of postnatal lethality $(\sim 50 \%$ survive to adulthood) in a hybrid background and in a complete loss of homozygous animals in an inbred genetic background (Ramirez-Solis et al. 1993). We are currently backcrossing the Hoxa-5 mutation into the inbred $129 / \mathrm{Sv}$ background to determine whether the penetrance of the phenotype is more pronounced.

Because of the genomic organization of the Hox cluster in vertebrates, it is difficult to assess whether the phenotype arises from a single gene mutation. One possibility is that owing to the close juxtaposition of genes within a cluster, the neo cassette might interfere with the correct expression of adjacent genes. The dynamic patterns of Hox gene expression are generated by a combination of positive and negative regulatory elements that differentially restrict the spatial, temporal, and tissue-specific domains (Zakany et al. 1988; Bieberich et al. 1990; Püshel et al. 1991; Tuggle et al. 1990; Whiting et al. 1991). The strong conservation of the genomic structure of the homeotic clusters between species such as human and mouse suggests that the precise overall organization of a given cluster is necessary for correct regulation and expression of the component genes. This is supported by the observation that long and interspersed transcripts, multiple promoters, shared regulatory re- 
gions, and differential splicing are features associated with Hox gene complexes (Boncinelli et al. 1991; Sham et al. 1992). Introduction of subtle mutations destroying the coding potential of specific Hox gene transcripts provides an important alternative method by which to assess the completely unique contribution of individual Hox genes (Hasty et al. 1991; Valancius and Smithies 1991; Ramirez-Solis et al. 1993).

A final possibility is that the paralogous genes Hoxb-5 and Hoxc-5 may compensate in part for loss of Hoxa-5 function. It is becoming increasingly clear that key regulatory molecules in development, such as growth factors or transcription factors, operate as networks of related family members and have overlapping spatial and temporal domains of expression. It will be interesting to compare the phenotypes of animals harboring single mutations with those carrying compound mutations of paralogous Hox genes.

To verify that the phenotype observed in the Hoxa-5 mutant mice results solely from a mutation at this locus, it will be necessary to complement the mutation using a Hoxa-5 transgene. However, one potential complication in using this approach is the necessity of defining the cis-acting regions required for the appropriate spatial and temporal regulation of Hoxa-5 gene expression. A 912-bp region located upstream of the first exon of the Hoxa-5 gene has been identified that is sufficient to direct lac $Z$ reporter gene expression in a discrete rostrocaudal region of the central nervous system (Zakany et al. 1988; Tuggle et al. 1990). However, the pattern of lacZ expression does not entirely recapitulate the endogenous Hoxa-5 expression pattern described by in situ hybridization analysis. An additional complication is that, as for most other Hox genes, multiple transcripts of different sizes are derived from the Hoxa- 5 locus. It has not been determined whether these alternate transcripts encode different functional forms of the Hoxa-5 protein as has been shown for the Hoxc-6 gene (Oliver et al. 1988). Northern analysis shows that the neo insertion introduced in the Hoxa-5 locus disrupts all four Hoxa-5 transcripts and therefore destroys the DNA-binding domain of any protein (or proteins) that might result from translation of these transcripts (Fig. 2).

In summary, mutation of the Hoxa-5 gene leads to developmental defects in those tissues that normally express the gene product. Moreover, the distribution of the abnormalities that characterize Hoxa-5 mutant mice is in general agreement with a combinatorial expression model, in which the expression of multiple Hox genes is required to specify axial identity, with mutation of a single component being sufficient to influence the regional specification in the developing embryo.

\section{Materials and methods}

Generation of germ-line chimeras

Embryo manipulations were carried out as described (Schwartzberg et al. 1989|. Ten to twelve ES cells from clonal ES cell lines carrying the Hoxa-5 mutation were injected into individual blastocysts, derived from either C57B1/6 or MF1 mice. Injected blastocysts were cultured in ES cell medium for l-2 hr and then transferred in groups of 6-10 into pseudopregnant female mice. Chimeric pups were identified by coat color chimerism. Chimeric males were bred to MFl females to test for germ-line transmission of the dominant agouti coat color marker. Heterozygous progeny carrying the mutated allele were identified following Southern blot analysis of tail DNA obtained from resultant $F_{1}$ agouti animals.

\section{Southern blot analysis}

Genomic DNA was prepared from either tail tissue or embryonic tissue as described in Hogan et al. (1986). Purified DNA was digested with the indicated restriction endonuclease, fractionated by electrophoresis through $0.8 \%$ agarose gels, blotted onto N-Hybond membrane (Amersham), and hybridized to the 850-bp BglII-HindIII ${ }^{32} \mathrm{P}$-labeled genomic probe containing the 3 '-untranslated region of the second exon of the Hoxa-5 gene.

\section{Northern analysis}

Total RNA from wild-type, heterozygous, and homozygous adult tissues was prepared as described in Chomczynski and Sacchi (1987), and Northern analysis was performed according to Maniatis et al. (1989). The 850-bp BgllI-HindIII genomic fragment containing the 3 -untranslated region of the second exon of the Hoxa-5 gene was used as a probe to detect endogenous Hoxa-5 mRNAs as well as mutated transcripts. In addition, blots were also hybridized with a mouse $\alpha$-actin probe (Minty et al. 1981 as well as with a neo probe.

\section{Skeletal analysis}

Specimens were prepared as described in McLeod (1980), except that staining with alcian blue $8 \mathrm{GS}$ and alizarin red $\mathrm{S}$ was performed for 7 days. Skeletons were then cleared in $2 \% \mathrm{KOH}$ before restaining with $0.015 \%$ alizarin red $\mathrm{S}$ in $1 \% \mathrm{KOH}$ for 24 hr to clearly identify ossified structures.

\section{Acknowledgments}

This work was supported by a grant from the National Cancer Institute of Canada (L.J.) and by the Raymond and Beverly Sackler Foundation (E.J.R.). L.J. is a scholar of the Canadian Medical Research Council (MRC) and the Cancer Research Society, Inc., and J.C. holds a scholarship from the Canadian MRC.

The publication costs of this article were defrayed in part by payment of page charges. This article must therefore be hereby marked "advertisement" in accordance with 18 USC section 1734 solely to indicate this fact.

\section{References}

Akam, M.E. 1987. The molecular basis for metameric pattern in the Drosophila embryo. Development 101: 1-22.

Balling, R., G. Mutter, P. Gruss, and M. Kessel. 1989. Craniofacial abnormalities induces by ectopic expression of the homeobox gene Hox1.1 in transgenic mice. Cell 58: 337-347.

Beddington, R.S.P., A.W. Püschel, and P. Rashbass. 1992. Use of chimeras to study gene function in mesodermal tissues during gastrulation and early organogenesis. CIBA Found. Symp. 165: 61-77.

Bieberich, C., M. Utset, A. Awgulewitsch, and F. Ruddle. 1990. Evidence for positive and negative regulation of the Hox3.1 gene. Proc. Natl. Acad. Sci. 87: 8462-8466. 
Boncinelli, E., A. Simeone, D. Acampora, and F. Mavilio. 1991. HOX gene activation by retinoic acid. Trends Genet. 7: 329 3-34.

Chisaka, O. and M.R. Cappecchi. 1991. Regionally restricted developmental defects resulting from targeted disruption of the mouse homeobox gene Hox1.5. Nature 350: 473-479.

Chisaka, O., T.S. Musci, and M.R. Capecchi. 1992. Developmental defects of the ear, cranial nerves and hindbrain resulting from targeted disruption of the mouse homeobox gene Hox1.6. Nature 355: 516-520.

Chomczynski, P. and N. Sacchi. 1987. Single-step method of RNA isolation by acid guanidium thiocyanate-phenol-chloroform extraction. Anal. Biochem. 162: 156-159.

Dony, C. and P. Gruss. 1987. Specific expression of the Hox 1.3 homeo box gene in murine embryonic structures originating from or induced by the mesoderm. EMBO J. 6: 2965-2975.

Gaunt, S.J., P.T. Sharpe, and D. Duboule. 1988. Spatially restricted domains of homeo-gene transcripts in mouse embryos: relation to a segmented body plan. Development (Suppl.) 104: 169-179.

Gaunt, S.P., P.L. Coletta, D. Pravtcheva, and P.T. Sharpe. 1990. Mouse Hox3.4: Homeobox sequence and embryonic expression patterns compared with other members of the Hox gene network. Development 109: 329-339.

Gehring, W.J. 1992. The homeobox in perspective. Trends Biochem. Sci. 17: 277-280.

Gruss, P. and Kessel, M. 1991. Axial specification in higher vertebrates. Curr. Opin. Genet. Dev. 1: 204-210.

Hasty, P., R. Ramirez-Solis, R. Krumlauf, and A. Bradley. 1991. Introduction of a subtle mutation into the Hox2.6 locus in embryonic stem cells. Nature 350: 243-246.

Hayashi, S. and M.P. Scott. 1990. What determines the specificity of action of Drosophila homeodomain proteins? Cell 63: 883-894.

Hogan, B., F. Costantini, and E. Lacy. 1986. Manipulating the mouse embryo. A laboratory manual. Cold Spring Harbor Laboratory, Cold Spring Harbor, New York.

Holland, P.W.H. and B.L.M. Hogan. 1988. Expression of homeo box genes during mouse development: A review. Genes \& Dev. 2: $773-782$.

Hunt, P. and R. Krumlauf. 1992. Hox codes and positional specification in vertebrate embryonic axes. Annu. Rev. Cell. Biol. 8: 227-256.

Jeannotte, L., J.C. Ruiz, and E.J. Robertson. 1991. Low level of Hox 1.3 gene expression does not preclude the use of promoterless vectors to generate a targeted gene disruption. Mol. Cell. Biol. 11: 5578-5585.

Jegalian, B.G. and E.M. De Robertis. 1992. Homeotic transformations in the mouse induced by overexpression of a human Hox3.3 transgene. Cell 71: 901-910.

Kaufman, M.H. 1992. The atlas of mouse development. Academic Press Limited, London, UK

Kaufman, T.C., M.A. Seeger, and G. Olsen. 1990. Molecular and genetic organization of the Antennapedia gene complex of Drosophila melanogaster. Adv. Genet. 27: 309-362.

Kessel, M. 1992. Respecification of vertebral identities by retinoic acid. Development 115: 487-501.

Kessel, M. and P. Gruss. 1991. Homeotic transformations of murine vertebrae and concomitant alteration of Hox codes induced by retinoic acid. Cell 67: 89-104.

Kuziora, M.A. and W. McGinnis. 1988. Autoregulation of a Drosophila homeotic selector gene. Cell 55: 477-485.

LeMouellic, H., Y. Lallemand, and P. Brûlet. 1992. Homeosis in the mouse induced by a null mutation in the Hox3.1 gene. Cell 69: 251-264.

Lewis, E.B. 1978. A gene complex controlling segmentation in
Drosophila. Nature 276: 565-570.

Lohnes, D., P. Kastner, A. Dierich, M. Mark, M. LeMeur, and P. Chambon. 1993. Function of retinoic acid receptor $\gamma$ in the mouse. Cell 73: 643-658.

Lufkin, T., A. Dierich, M. LeMeur, M. Mark, and P. Chambon. 1991. Disruption of the Hox1.6 homeobox gene results in defects in a region corresponding to its rostral domain of expression. Cell 66: 1105-1119.

Lufkin, T., M. Mark, C. P. Hart, P. Dollé, M. LeMeur, and P. Chambon. 1992. Homeotic transformation of the occipital bones of the skull by ectopic expression of a homeobox gene. Nature 359: 835-841.

Maniatis, T., E.F. Fritsch, and J. Sambrook. 1989. Molecular cloning: A laboratory manual. Cold Spring Harbor Laboratory Press, Cold Spring Harbor, New York.

Marshall, H., S. Nonchev, M.H. Sham, I. Muchamore, A. Lumsden, and R. Krumlauf. 1992. Retinoic acid alters hindbrain Hox code and induces transformation of rhombomeres $2 / 3$ into a 4/5 identity. Nature 360: 737-741.

McGinnis, W. and R. Krumlauf. 1992. Homeobox genes and axial patterning. Cell 63: 969-976

McLain, K., C. Schreiner, K.L. Yager, J.L. Stock, and S.S. Potter 1992. Ectopic expression of Hox2.3 induces craniofacial and skeletal malformations in transgenic mice. Mech. Dev. 39: 3-16.

McLeod, M.J. 1980. Differential staining of cartilage and bone in whole mouse fetuses by alcian blue and alizarine red S. Teratology 22: 299-301.

Merrill, V.K.L., R.J. Diederich, F.R. Turner, and T.C. Kaufman 1989. A genetic and developmental analysis of mutations in labial, a gene necessary for proper head formation in Drosophila melanogaster. Dev. Biol. 135: 376-391.

Minty, A.J., M. Caravatti, B. Robert, A. Cohen, P. Daubas, A. Weydert, F. Gros, and M.E. Buckingham. 1981. Mouse actin messenger RNAs. Construction and characterization of a recombinant plasmid molecule containing a complementary DNA transcript of mouse $\alpha$-actin mRNA. J. Biol. Chem. 256: 1008-1014.

Odenwald, W.F., C.F. Taylor, F.J. Palmer-Hill, V. Friedrich Jr., M. Tani, and R.A. Lazzarini. 1987. Expression of a homeo domain protein in noncontact-inhibited cultured cells and postmitotic neurons. Genes \& Dev. 1: 482-496.

Odenwald, W.F., J. Garbern, H. Arnheiter, E. Tournier-Lasserve, and R.A. Lazzarini. 1989. The Hoxl.3 homeo box protein is a sequence-specific DNA-binding phosphoprotein. Genes \& Dev. 3: 158-172.

Oliver, G., C.V.E. Wright, J. Hardwicke, and E.M. De Robertis 1988. Differential antero-posterior expression of two proteins encoded by a homeobox gene in Xenopus and mouse embryos. EMBO J. 7: 3199-3209.

Otting, G., Y.Q. Qian, M. Billeter, M. Müller, M. Affolter, W. J. Gehring, and K. Wüthrich. 1990. Protein-DNA contacts in the structure of a homeodomain-DNA complex determined by nuclear magnetic resonance spectroscopy in solution. EMBO /. 9: 3085-3092.

Papalopulu, N., J.D.W. Clarke, L. Bradley, D. Wilkinson, R. Krumlauf, and N. Holder. 1991. Retinoic acid causes abnormal development and segmental patterning of the anterior hindbrain in Xenopus embryos. Development 113: 11451158 .

Pollock, R.A., G. Jay, and C.J. Bieberich. 1992. Altering the boundaries of Hox3.1 expression: Evidence for antipodal gene regulation. Cell 71: 911-923.

Püshel, A., R. Balling, and P. Gruss. 1991. Separate elements cause lineage restriction and specify boundaries of Hox1.1 expression. Development 112: 279-287. 
Ramirez-Solis, R., H. Zheng, J. Whiting, R. Krumlauf, and A. Bradley. 1993. Hoxb-4 (Hox2.6) mutant mice show homeotic transformation of a cervical vertebra and defects in the closure of the sternal rudiments. Cell 73: 279-294.

Schughart, K., C. Kappen, and F.H. Ruddle. 1989. Duplication of large genomic regions during the evolution of vertebrate homeobox genes. Proc. Natl. Acad. Sci. 86: 1-5.

Schwartzberg, P.L., S.P. Goff, and E.J. Robertson. 1989. Germline transmission of a c-abl mutation produced by targeted gene disruption in ES cells. Science 246: 799-804.

Scott, M.P. 1992. Vertebrate homeobox gene nomenclature. Cell 71: 551-553.

Sham, M.-H., P. Hunt, S. Nonchev, N. Papalopulu, A. Graham, E. Boncinelli, and R. Krumlauf. 1992. Analysis of the murine Hox2.7 gene: Conserved alternative transcripts with differential distributions in the nervous system and the potential for shared regulatory regions. $E M B O F$. 11: 1825-1836.

Simeone, A., D. Acampora, V. Nigro, A. Faiella, M. D'Esposito, A. Stornaluolo, F. Mavillo, and E. Boncinelli. 1991. Differential regulation by retinoic acid of the homeobox genes of the four HOX loci in human embryonal carcinoma cells. Mech. Dev. 33: 215-227.

Tuggle, C.K., J. Zakany, L. Cianetti, C. Peschle, and M.C. Nguyen-Huu. 1990. Region-specific enhancers near two mammalian homeo box genes define adjacent rostrocaudal domains in the central nervous system. Genes \& Dev. 4: 180-189.

Valancius, V. and O. Smithies. 1991. Testing an "in-out" targeting procedure for making subtle genomic modifications in mouse embryonic stem cells. Mol. Cell. Biol. 11: 14021408.

Wakimoto, B.T. and T.C. Kaufman. 1981. Analysis of larval segmentation in lethal genotypes associated with the Antennapedia gene complex in Drosophila melanogaster. Dev. Biol. 81: 51-64.

Whiting, J., H. Marshall, M. Cook, R. Krumlauf, P. Rigby, D. Stott, and R. Allemann. 1991. Multiple spatially-specific enhancers are required to reconstruct the pattern of Hox2.6 gene expression. Genes \& Dev. 5: 2048-2059.

Zakany, J., C.K. Tuggle, M.D. Patel, and M.C. Nguyen-Huu. 1988. Spatial regulation of homeobox gene fusions in the embryonic central nervous system of transgenic mice. Neuron 1: 679-691.

Zhao, J.J., R.A. Lazzarini, and L. Pick. 1993. The mouse Hox1.3 gene is functionally equivalent to the Drosophila Sex combs reduced gene. Genes \& Dev. 7: 343-354. 


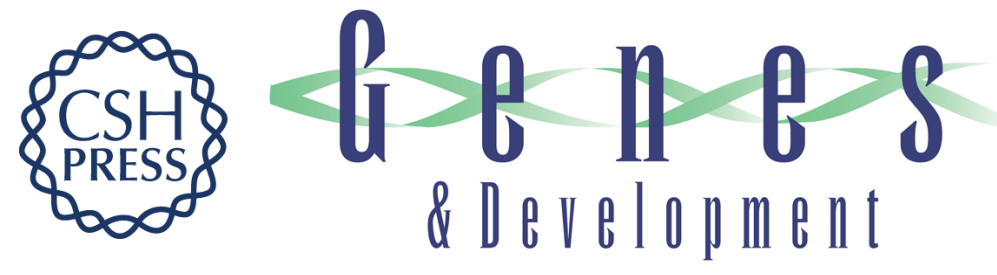

\section{Specification of axial identity in the mouse: role of the Hoxa-5 (Hox1.3) gene.}

L Jeannotte, M Lemieux, J Charron, et al.

Genes Dev. 1993, 7:

Access the most recent version at doi:10.1101/gad.7.11.2085

References This article cites 52 articles, 18 of which can be accessed free at:

http://genesdev.cshlp.org/content/7/11/2085.full.html\#ref-list-1

License

Email Alerting

Service

Receive free email alerts when new articles cite this article - sign up in the box at the top right corner of the article or click here.

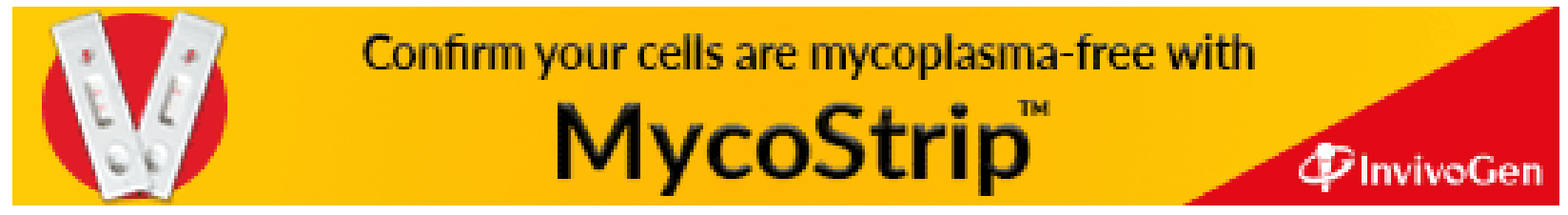

